\title{
LA DYNAMIQUE DE L'IMAGINATION DE JULIEN GRACQ DANS LE RIVAGE DES SYRTES
}

\author{
Maria Tereza de Freitas
}

\begin{abstract}
Chez Gracq, la description. est la rêverie même de l'homme devant le monde, la merveilleuse inconsistance du vécu: il n'y a que cette suite de moments privilégiés, singuliers, étranges, de paysages pas tout à fait réels. Rien n'est jamais complètement vrai, puisqu'il n'existe que l'irréalité du monde." (1)
\end{abstract}

Considéré comme un des romans du "monde réel" selon l'expression de Louis Aragon (2), Le Rivage des Syrtes (3) semble être à la limite de ce problème crucial de toute la littérature qu'est la distinction entre le réel et l'imaginaire, distinction "impossible à soutenir" selon Todorov (4). Seulement, dans ce roman ce problème est encore plus complexe. Il ne s'agit pas de savoir à quel point les catégories du réel sont mises en cause par l'intervention de l'imaginaire. Il s'agit plutôt de considérer la mise en question de ce qui est donné comme réel dans le roman, en tant que représentatif de lui-même et non du monde; ce n'est pas le réel lui-même, mais plutôt une certaine conception du réel qui est mise en cause dans ce roman. Fondé sur un imaginaire surréaliste, qui n'accepte pas le vraisemblable pour vrai, mais qui cherche ses propres lois de cohérence (s' il y en $a$. .) dans l'inconscient, c'est un roman qui tente de vérifier les rapports du conscient avec l'inconscient. Dans un climat de rêve, dans une atmosphère surréelle, un personnage moulé sur le réel agit dans un espace inspiré du réel et donné pour réel dans le roman: dans quelle

(1) - Ariel DENIS, "La description romanesque dans l'oeuvre de Gracq", in Revue d'Esthétique, tome 22-1969, p. 164.

p. 169 .

(2) - Cf. Cl. ABASTADO, Le Surréalisme. Paris, Hachette, 1975,

(3) - Paris, Corti, 1951. p. 31 .

(4) - Cf. Introduction à la littérature fantastique. Paris, Seuil 1970.

Língua e Literatura, (14), 1985. 
mesure rêve et réalité se confondent dans la conscience d'Aldo, personnage-narrateur, aussi vraisémblable que possible puisqu'il dit "je", et qu'il raconte une histoire, son histoire?

\section{Le réel et l'imaginaire}

De toute évidence, ce roman ne correspond pas aux récits authentiques, que réclame Breton (5); Aldo est un personnage fictif, sorti de l'imaginaire de Julien Gracq, le héros vraisemblable dont les actions et les réactions sont plus ou moins prévues, et s'il raconte sa vie, son expérience personnelle, il ne s'agit aucunement déune aven. ture vécue; il a, derrière lui, un auteur qui dispose et qui décide de ses mouvements et de son destin, qui crée. Nous avons affaire à une oeuvre de fiction, qui se reconnaît comme telle et qui raconte une histoire imaginée par l'auteur. Ce qui n'empêche qu'il s'agit d'un roman inspiré du monde réel, qui s'inscrit dans la catégorie de la représentation, qui obéit à une certaine cohérence interne, et qui appelle donc une lecture plus ou moins référentielle. Récit "vraisemblable" à "motivation implicite", selon la terminologie de G. Genette (6), où les unités narratives s'enchaînent par une détermination causale apparemment naturelle, Le Rivage des Syrtes feint de copier la réalité extérieure, ne mettant pas en question les lois du monde représenté, ni celles de la fiction (7). Aldo, le personnage-narrateur, raconte comment il arrive à changer le destin d'une nation endormie, Orsenna, en réactivant une guerre oubliée contre un pays ennemi, le Farghestan. Cependant, le sujet du roman n'est pas l'anecdote en elle-même, ni non plus lees événements en eux-mêmes, mais plutôt le processus par lequel l'inconscient d'un personnage devient réel: Aldo raconte, sans dissocier rêve et réalité, des aventures qui lui sont arrivées; tout en étant aux prises avec le monde extérieur, il vit dans un monde intérieur Le dynamisme de l'action ne se trouve pas dans les événements racontés, car c'est un monde essentiellement inactif, mais il naît plutôt des possibles imaginaires suggérés par ces événements et activés dans l'imagination d'Aldo; on assiste ainsi à la création d'un monde invisible mais qui contient les significations du monde réel présenté: une série de personnages et de lieux énigmatiques provoque l'irruption de l'imaginaire, qui demeure toujours dans une

(5) - Dans "Introduction au discours sur le peu de réalité" in Point du Jour, N.R.F., coll. Idées, p. 9.

(6) - "Vraisemblable et motivation" in Communications 11. Paris. Seuil, 1968, p. 17.

(7) - Pour une analyse plus détaillée du degré de représentativité de ce roman, voir notre Dissertation de Maîtrise, "Fiction et surréalisme dans Le Rivage des Syrtes de J. Gracq", 1974, pp. 187 à 263é 
zone obscure, reflet de la réalité extérieure, elle aussi obscure et mystérieuse. L'entreprise d'Aldo consiste dans le déchiffrement d'une réalité qui, comme on a déjà signalé, se trouve "située au-delà de celle qui est évidente, cette dernière ne jouant que le rôle de masque par rapport à un dévoilement ultérieur" (8). Au lieu de "masque" nous dirions plutôt stimulus: le destin d'Orsenna sera accompli d'abord dans l'imaginaire d'Aldo. Les distanciations opérées dans l'imagination à partir du réel conservent trop d'équivalences implicites avec celui-ci, et présentent une solution de continuité trop rigoureuse, ce qui fait que la logique initiale du réel ne se trouve jamais véritablement contredite ni niée par son prolongement dans l'imaginaire. Il ne s'agit donc pas de deux mondes tifférents - l'un réel et l'autre imaginaire -, l'un interférant dans l'autre, mais, d'une seule réalité envisagée dans un champ plus vaste, où réel et imaginaire se fondent, une réalité quì, par son caractère incomplet et énigmatique, se complète dans l'imaginaire et forme avec lui l'univers réel du roman. Le réel est donc, non pas simplement le monde référentiel, mais la somme de l'apparent plus le latent, l'embryonnaire, le possible, ce qui n'est pas encore complètement réalisé, mais en voie de l'être: la réalité implique l'imaginaire. Le Rivage des Syrtes représente le passage du possible à son actualisation: la coupure ne se fait pas entre ce qui est rêvé, phantasmé, et ce qui est réel, mais entre ce qui est présent et ce qui va devenir présent et qui est en train de se réaliser; c'est là où l'imaginaire joue son rôle: il est pour Gracq le grand essai à travers lequel le possible se réalise. L'auteur cherche ainsi à abattre les cloisons de la raison, dans lesquels s'abritent d'ordinaire les lois qui régissent le système des catégories de la représentation et qui établissent la distinction entre le tangible et le rêve. Cette distinction est pour lui fausse, arbitraire, et il s'agit de trouver ce "point de l'esprit" où, comme le voulait Breton, les deux mondes "cessent d'être perçus contradictoirement" (9). Certes, il le fait par un effort raisonné - d'où le caractère "réaliste" de sa fiction - mais il n'en prend pas moins en compte le pouvoir de l'imaginaire dans l'univers romanesque "réel" qu'il crée. En voici quelques exemples:

A plusieurs reprises, Aldo parle de son aventure comme d'un "songe vécu"; près d'accomplir l'acte qui le mènera au bout du chemin, il observe l'ami qu'il entraîne avec lui:

"ce visage que j'emportais dans mon songe vivait comme il n'avait jamais vécu" (p. 214):

(8) - Anne Fabre-Luce, "Dialectique de l'imaginaire dans Le Rivage des Syrtes". in Revue des Sciences Humaines, XXXIII, Janvier-mars, 1968, p. 129.

(9) - Manifestes du Surréalisme. Paris, Gallimard, 1972, pp. 76-77 
l'antinomie rêvé/vécu disparaît. C'est ce que traduit également la phrase qui décrit la transgression de la chambre des cartes:

"Il me semblait que nous venions de pousser une de ces portes qu'on franchit en rêve." (p. 204):

le modalisateur "il me semblait" exclut la vrai possibilité du rêve et montre bien qu'il s'agit d'un événement tenu pour réel dans la cohérence interne du roman; cependant il est perçu comme un rêve, car c'est en effet un rêve qui devient réel: il avait si souvent franchi cette porte dans son imagination, il avait si longemps rêvé de cette transgression de la chambre interdite, que maintenant réel et imaginaire se confondent dans sa perception des choses.

A Marino, le seul personnage qui tienne encore à ne pas rêver - ce qui dans ce monde complexe équivaut à ne vivre qu'à moitié --, et pour qui "l'imagination est de trop" ( $p$. 47), Aldo s'efforce de faire comprendre la réalité du monde imaginaire:

"J'avais hâte d'éprouver sur Marino la réalité même, pourtant indubitable, de cette apparition suspecte." (p. 42)

Cet effort montre l'évidence de la confusion des deux mondes: la réalité est tellement énigmatique qu'elle présente les caractéristiques du rêve. Le soin même que Marino accorde à empêcher les rêves, fait preuve de leur importance: il veut cacher une chose dangereuse, donc réelle; la réplique d'Aldo est très juste:

"Alors, je vous croirai. un. peu romanesque. Je ne pensais pas que la vie à l'Amirauté cachait tant de fantastique. J'ai peur que vous n'en ajoutiez un peu." ( $p .45$ ).

A plusieurs reprises, les descriptions oscillent entre le réel et l'imaginaire: on passe aisément d'un plan à l'autre, car l'interpénétration est totale. Ainsi, par exemple, à Vezzano, lorsque Aldo et Vanessa arrivent enfin à voir le Tängri - ce mystérieux volcan qui exerce une étrange fascination sur les personnages - c'est dans une ambiance de songe, presque irréelle, qu'ils le perçoivent:

"Son lever d'astre sur l'horizon ne parlait pas de la terre, mais plutôt d'un soleil de minuit, de la révolution d'une orbite calme qui l'eût ramené à l'heure dite des profondeurs lavées à l'affleurement fatidique de la mer. Il était là. Sa lumière froide rayonnait comme une source de silence, comme une virginité déserte et étoilée. (...) La lumière de la lune tirait vaguement de l'ombre la cime énigmatique pour l'y replonger 
aussitôt, la faisait palpiter irréellement sur la mer éfacée" (p. 151);

on a l'impression que dans ces moments contemplatifs le monde réel n'existe pas; même le temps disparaît, comme l'observe le personnage lui-même. Tout d'un coup, sans aucune transition, des sensations extérieures se font sentir:

"Enfin, il fit nuit tout à fait, le froid nous transperģa. Je relevai Vanessa sans mot dire elle s'appuya à mon bras tout lourde" (ibid.)

Cependant, le songe ne se défait pas comme l'on pourrait s'y attendre; il se poursuit au contraire dans le réel, et les deux fusionnent à tel point qu'on n'arrive plus à discerner ce qui réel de ce qui ne l'est pas:

"Nous marchions la tête vide, les yeux douloureux de cet excès de fixité, les jambes molles ( . .) Il me semblait que sur cette journée de douce et caressante chaleur avait comme un vent descendu des champs de neige, si lustral et si sauvage que jamais mes poumons qu'il avait mordus n'en pourraient épuiser la pureté mortelle, et comme pour en garder encore l'étincellement dans mes yeux et la saveur froide sur ma bouche, sur le sentier ébouleux, malgré moi, je marchais la tête renversée vers le ciel plein d'étoiles." (ibid).

Le passage qui suit le sermon de Noël à l'église Saint-Damase, constitue également un excellent exemple de la fragilité des limites entre le réel et l'imaginaire dont le roman fait preuve: la frontière qui sépare le contenu du sermon - qui n'exprime que le réel latent, déjà vécu en imagination par Aldo - des images concrètes qui entourent le personnage, tend à disparaître. La nuit "réelle" dans laquelle marche Aldo, devient la "nuit promise" du sermon; soudain, comme si c'était naturel, les deux plans s'associent:

"Je sombrais insensiblement dans le sommeil. L'image de Marino assis dans son bureau de l'Amirauté passait par instants devant mes yeux (. .); il oscillait devant moi au rythme de la barque comme un homme qui marche sur les eaux, pareil à un pantin dérisoire, puis les oscillations se firent moins amples; un instant le visage se tint devant moi dans une immobilité pesante, et je sentis plonger dans les miens ses yeux taciturnes et fixes, mais aussitôt je $\mathrm{m}^{\prime}$ endormis." (pp. 180-181)

Rêve ou souvenir? Réel ou imaginaire? Une fusion des deux, sans aucun doute. 
Le même mouvement peut être observé dans les descriptions des ruines de Sagra (p. 71), de Maremma (pp. 82 et 83), de la forteresse reconstruite (p. 130), du Tängri vu du bateau (pp. 215 et 216), où des mots tels que: "songe", "irréel", "rêve", alternent sans cesse tantôt avec des sensations extérieures "réelles" (bruits, odeurs, regards sur la nature), tantôt avec des descriptions d'objets concrets. A titre d'illustration - bien que tout le passage soit significatif - voici la phrase qui clôt la description du Tängri, vu de très près:

"Le silence autour de cette apparition qui appelait le cri angoissait l'oreille, comme si l'air tout à coup se fût révélé opaque à la transmission du son, ou encore, en face de cette paroi constellée, il évoquait la chute nauséeuse et molle des mauvais rêves où le monde bascule, et où le cri au-dessus de nous d'une bouche intarissablement ouverte ne nous rejoint plus." (p. 216)

Des sensations concrètes sont parfois perçues comme en rêve:

"Les mots résonnèrent dans ma tête, insignifiants comme des cailloux qu'on agite dans une boîte creuse. Puis un vide se forma sous mon estomac, et je me sentis envahir par cette sensation nauséeuse du rêve où l'on sent au bord du gouffre un garde-fou céder pouce par pouce sous les doigts." (p. 264).

De même que parfois des mots percent le réel et parviennent à Aldo comme ceux de quelqu'un qui rêve, de "quelqu'un qui parle au milieu de son sommeil" (p. 307). Rêve et réalité se confondent; celle-ci est perçue comme le prolongement de celui-là .

\section{Les perturbations du réel}

Cette interpénétration se réalise pleinement dans l'atmosphère en quelque sorte indéfinissable où baigne le récit: les limites de la sensibilité du personnage se perdent dans les horizons illimités de l'imagination et du rêve, et nous nous trouvons devant une atmosphère surnaturelle sans qu'il y ait du surnaturel proprement dit. Comme l'a très justement observé Ariel Denis, "les romans de Julien Gracq... sont d'éternelles promenades, de perpétuelles rêveries sur le monde, desquelles naît ce sentiment profond de mystère sans que rien ne soit mystérieux, d'étrangeté sans que rien ne soit étrange, d'irréalité et pourtant ce n'est que le réel qui est décrit." (10). En effet, c'est bien

(10) - "La description romanesque dans l'oeuvre de Gracq", in Revue d'Esthétique, tome 22, 1969, p. 1656. 
dans la création d'une atmosphère d'irréalité et d'étrangeté qu'on peut trouver cette espèce de progression insolite qui met en cause l'intelligibilité rationnelle du réel dans ce roman. Deux éléments semblent contribuer fondamentalement à créer cette atmosphère: le fantomatique et le mystérieux.

\section{a) Le fantomatique}

Cet élément s'y manifeste sous plusieurs formes.

Considérons d'abord l'éclairage des lieux: on s'aperçoit que l'ombre et la pénombre, le brouillard et la brume prédominent, ce qui produit un éclairage ambigu, qui provoque l'imprécision des contours et suscite des visions pas très nettes; sous cet éclairage de rêve où rien n'est clair, les limites matérielles du monde s'effacent: les contours du réel s'estompent, la vision n'est plus tributaire d'objets bien délimités et elle s'ouvre sur des perspectives plus vastes; le réel prend les dimensions du songe et les "fantômes" apparaissent. Ces lieux acquièrent alors un aspect fantomatique. Quand Aldo arrive à l'Amirauté, qu'il trouve enveloppée d'un "brouillard épaissi", il aperçoit quelques "fantômes de bâtiments" et la silhouette de Marino "qui ba. lançait un fanal" (p. 19); aussi bien Marino que l'Amirauté apparaissent comme des fantômes, sortis de "cette fantasmagorie de brume", le mot de "silhouette" se prêtant bien à désigner une figure un peu onirique, produite par le jeu d'ombre et lumière, de clair et obscur. Voici comment le personnage-narrateur résume sa description de ce lieu:

"Ainsi surgie des brumes fantomatiques de ce désert d'herbes, au bord d'une mer vide, c'était un lieu singulier que cette Amirauté. (..) le brouillard grandissait les contours d'une espèce de forteresse ruineuse." (pp. 20 et 21)

L'insistance sur le mot "brouillard" (il revient sept fois en deux pages) produit un effet de lourdeur dans l'atmosphère et souligne le manque de contours, d'autant plus que la pénombre rejoint bientôt le brouillard et rend encore plus forte l'impression d'irréalité; dans cette atmosphère, même les personnages sont comparés à des fantômes du passé:

"Sous cet éclairage théâtral et faible, un halo de brume trainait... autour des visages. .; la contrainte hésitante et raidie d'une première rencontre ajoutait encore à la bizarre impression d'irréel qui m'envahissait (); les visages des con- 
vives devenaient de pierre, retrouvaient un instant le profil dur et le masque austère des vieux portraits de l'âge heroïque pendus aux palais d'Orsenna." (p. 22)

Une fois établi ce climat, le fantomatique ne quittera plus jamais l'Amirauté:

“ la forteresse lavée des regards indifférents reprenait les dimensions du songe. Mes pieds légers et assourdis erraient dans les couloirs à la manière des fontômes dont le pas, à la fois hésitant et guidé, réapprend un chemin" (p. 35).

A travers le brouillard, Aldo va apercevoir "l'ombre à peine distincte d'un petit bâtiment" glissant sur la mer, gu'il appellera plus tard "navire-fontôme" (p. 44) Après sa reconstruction, la forteresse est comparée à "un fantôme sous son suaire" (p. 213).

La scène de la mort de Marino se passe dans une ambiance tout à fait onirique, presque surnaturelle: éclairage, brouillard et fantôme se fondent en une image de rêve lugubre:

"Il y avait dans l'oeil de Marino - était-ce un reflet de cet éclairage fantomatique? - quelque chose de fixe et de lugubre ( .) La lanterne posée entre nous à terre tirait à peine les visages du halo de vapeur; nos ombres allongées s'arquaient en se perdant très haut sur les voûtes (. ). Le silence se faisait pesant. La flamme de la lanterne pétillait et charbonnait dans l'air nauséeux, des ombres douteuses grouillaient sur les voûtes souillées ( .) La nuit était très sombre mais.. à travers le brouillard bleuâtre, montait un souffle d'humidité pénétrante ( ): toutes choses reposaient dans l'intimité noire d'une cloche de ténèbres ( .) Tapi contre la pierre, la tête dans les épaules, mon coeur se suspendit à un instant de silence surnaturel, puis avec un bruit flasque, un corps giffla lourdement les eaux calmes" (pp. 273, 274, et 275).

Lors de la recherche du corps de Marino, brouillard et fantôme s'associent encore une fois:

". parfois un homme surgissait du brouillard comme un fantôme glissant sur les eaux tranquilles et huileuses." (p. 276);

et finalement, le capitaine est comparé à un "génie engourdi de la terre" qui hantait l'Amirauté (p. 276). 
On peut observer la même atmosphère de songe à Sagra, lieu sinistre, hanté de fantômes:

\author{
"J'avançais dans un froissement d'osselets qui faisait \\ vivre sinistrement ces solitudes" (p. 69)
}

Aldo y marche "derrière une brume de mirage", comme un fantôme guidé par "un magnétisme secret" (p. 69); la pénombre, provoquant la rêverie, fait que tout dans ces ruines évoque des fantômes ou des âmes damnées (cf. p. 71). Dans cette atmosphère de rêve et d'imprécision, le personnage aperçoit sous "les restes de lumière d'un reflet de métal" le même bateau-fantôme qu'il avait vu à l'Amirauté, et "la silhouette d'un homme avec quelque chose de singulièrement souple dans sa démarche" (p. 72 et 73). Tout se passe comme dans un rêve, dans l'obscurité de la lumière diffuse, aux contours indéfinissables qui nous font penser plutôt à une apparition fantomatique qu'à un élément réel:

"L'ombre me dérobait déjà la silhouette à peine entrevue, brouillant une impression presque indéfinissable.." (p. 73).

Maremma, dans sa première description, apparaît elle aussi comme une figure de rêve, enveloppée de brouillard et de "lumière diffuse", provoquant un "sentiment d'irréalité" (p. 82). Et bientôt les fantômes y font également leur apparition:

"Parfois, au détour d'une rue.. apparaissait une femme de pêcheur ( . .): elle passait près de moi silencieusement comme un fantôme errant de ville morte $(\ldots)$. La vie voletait par les rues effacées comme un lambeau de ténèbres oublié dans le plein jour" (pp. 159-160).

À côté de ces lieux insolites, il y en a d'autres que, par leur bipolarité de sens, on pourrait appeler des lieux privilégiés. Dans ces endroits, eux aussi hantés par des fantômes, il ne s'agit pas seulement d'un paysage onirique dans lequel objets et personnages, vus à travers des contours indéfinissables, nous apparaissent comme des fantômes sans nom; ici, on voit aussi des fantômes précis, liés ou bien au passé - présence des morts - ou bien au futur - présages maléfiques.

Vezzano, île équivoque et lugubre, située à mi-chemin entre les terres d'Orsenna et le Farghestan, est un lieu de partage et d'échange hanté de mort, mais aussi "haut-lieu", terrasse pour un regard vers le futur, d'où l'on peut voir le Tängri. Dans cette île funèbre, à un 
"passé de sang versé et de richesses barbares" (p. 141), rêve et réalité s'affrontent:

"il me semblait... que l'horizon devant notre étrave s'orientait et se creusait mystérieusement" (p. 145)

La description abonde en expressions qui traduisent de mauvais pressentiments: "solitude malveillante et hargneuse" (p. 145), "la solitude et l'hostilité de cette Cythère morne" (p. 146), "mauvais présage", "île mal famée vaguement suspecte" (p. 149). Les allusions à son "passé funèbre" l'entourent d'un climat fantomatique:

"Ces cris sauvages et désolés des oiseaux de mer qui .. froidissaient cette ombre spectrale, ces roches nues d'un blanc gris d'ossements, et le souvenir de ce passé funèbre, jetaient un nuage inattendu sur cette mer de fête." (p. 146)

C'est comme si, dans ces mauvais pressentiments, on sentait la présence des fantômes du passé qui hantent l'île:

"On eût dit qu'avant l'heure les fantômes du soir se hâtaient de reprendre possession de la lande. ( .), la pensée me vint un instant que l'île était habitée, et qu'une silhouette allait surgir de ces roches qui donnerait corps à (la) fièvre (de Vanessa) et à mon malaise." (p. 149)

La "chambre vide" est un autre de ces lieux privilégiés. Gracq lui-même nous définit ce motif:

"J'ai toujours l'impression dans ce cas-là que l'être absent surgit du rassemblement des objets familiers autour de lui, de l'air confiné qu'il a respiré, de cette espèce de suspens des choses qui se mettent à rêver de lui tout haut, avec une force de conviction plus immédiate encore que sa présence ( ). Il s'agit peut-être pour moi de quelqu'un qui devrait être là.. et qui n'y est pas." (11).

Dans la chambre vide, on sent des présences indéfinissables, on a l'impression que les objets se raniment et que les dimensions du temps s'effacent, le passé se confondant avec l'avenir Il y en a trois, de ces pièces: la chambre des cartes, la chambre de Vanessa et la cabine de Marino dans le bateau.

(11) - J. GRACQ, "Les yeux bien ouverts" dans Préférences, Paris. Corti, 1961, pp. 65 et 66. 
La chambre des cartes, cette espèce d'île au centre de la forteresse, "tendue comme un piège" (p. 31), piéce sommairement meublée, avec des "murs nus" (p. 30), où "traîne une atmosphère lourde" (p. 31 ), est le lieu idéal où l'esprit peut s'abandonner à la rêverie. Le caractère insolite de cette salle est immédiatement dénoncé: elle n'est pas dans l'ordre, elle a quelque chose de dérangeant, de perturbateur. L'atmosphère fantomatique est vite suggérée: Aldo entre dans cette chambre comme dans un sépulcre:

$$
\begin{gathered}
\text { " la fraîcheur de sépulcre tombait en nappe sur mes } \\
\text { épaules, - j'entrais dans la chambre des cartes" (p. 29) }
\end{gathered}
$$

Désormais, on aura toujours l'impression que la pièce est peuplée par d'étranges présences qui la hantent et la surveillent, comme des fantômes du passé:

"En faisant grincer les gonds sur cette solitude surveillée.. je ne pouvais m'empêcher de reessentir chaque fois le léger choc qu'on éprouve à pousser à l'improviste la porte d'une pièce apparemment vide sur un visage soudain plus sinistre que celui d'un aveugle. " (p. 30)

Un double mouvement s'établit: d'une part, les choses sollicitent l'imagination, et l'autre part, l'imagination se projette sur les choses. D'un côté, le réel est étrange, par son aspect insolite: la disposition des objets suggère la présence de forces occultes, suscite de "mauvais songes" ( $\mathrm{p}$. 77); Aldo est comme hypnotisé par cette chambre: ce "lieu attirant" le magnétise, s'empare de lui; quand il s'assoit devant les cartes il est "enchaîné là comme par un charme" (p. 31); le mot d'hypnotisme même est employé:

“ je demeurais là parfois des heures, englué dans une immobilité hypnotique d'où ne me tirait pas même le fourmillement de mes paumes." (p. 32).

D'un autre côté, il y a aussi une projection de la conscience dans le monde extérieur: l'imagination agit sur les données sensibles du réel et les transforme en surnaturelles:

"Un bruissement léger semblait s'élever de cette carte, peupler la chambre close et son silence d'embuscade. Un craquement de la boiserie parfois me faisait lever les yeux, mal à l'aise, fouillant l'ombre. comme si j'avais guetté malgré moi, dans le silence de cloître, quelque chose de mystérieusement éveillé." (p. 32). 
Sous la force de l'imagination, les objets se raniment, l'abstrait devient concret: le drapeau de soie rouge - emblème d'Orsenna - est "une large tache de sang frais éclaboussant le mur" (p. 31); ce qu'Aldo regarde sur la table, ce ne sont pas des cartes, mais "les terres stériles des Syrtes" elles-mêmes ( $p$. 31); la ligne continue qui marque la frontière interdite entre Orsenna et le Farghestan, ce tracé "curieusement abstrait" finit par "s'imprégner d'un caractère de réalité bizarre' et devient un "interdit magique" Les limites entre le rêve et le réel s"effacent complèment:

“ les murs de la salle continuaient à bouger légèrement, comme si le songe eût résisté à crouler autour de cette chambre mal défendue" ( $p$. 77).

Le sentiment de viol qui accompagne la pénétration dans la chambre des cartes (quand Marino le surprend, Aldo a l'impression d'être pris en "flagrant délit"), semble lui donner un sens prémonitoire: cette première expérience de la transgression représente un prélude à la transgression majeure qui aura lieu plus tard, lorsqu' Aldo dépassera la frontière d'alarme; d'autant plus que c'est dans cette chambre que son regard, attiré au-delà de la zone de sécurité, dépasse sur la carte la ligne rouge. Ce même sentiment de viol s'emparera d'Aldo au moment où, avant de partir pour la Croisière qui le mènera à dépasser vaaiment cette ligne imaginaire, il entre une dernière fois dans la salle pour "voler" les cartes:

\footnotetext{
"J'appartenais maintenant à ce viol sans retour; je sortis à reculons de la pièce condamnée, tout pâle, tenant le rouleau de cartes serré contre moi, comme un voleur de tombeau que pousse la faim nue, qui sent rouler sous ses doigts les pierreries de rêve, et déjà la force du sortilège faire cailler lentement son sang." (p. 193);
}

ce qui frappe particulièrement dans ce passage, c'est l'image de "voleur de tombeau": il ne se sent pas comme un voleur ordinaire, car, en fait, il a le droit de prendre les cartes, il ne les vole à personne. Mais, on n'a pas affaire à une chambre normale; il s'agit de la "chambre vide" où l'on sent une présence absente - "quelqu'un qui devrait être là et qui n'y est pas" Quoiqu'il s'en tienne au seul niveau de l'image, le caractère surnaturel de cette chambre nous saisit brutalement dans ce passage: on sent comme si un lieu sacré avait été violé.

La chambre de Vanessa, cette "immense pièce aux murs nus", éclairée à peine d'une "lumière faible" elle aussi une chambre vide, 
est un autre noyau d'insolite; son vide provoque une sentation de malaise chez Aldo:

"L'espace désoeuvré de la chambre derrière moi me raidissait, pesait à mes épaules comme un thêâtre vide." (p. 99).

L'analogie de cette pièce avec la chambre des cartes est à remarquer; ici comme là, on sent dès le début des présences invisibles qui peuplent la chambre et la raniment:

"Un faible et profond murmure entrait par les fenêtres, peuplait maintenant le silence revenu et faisait vivre sourdement autour de nous chambre vide." (p. 104);

sous l'éclairage voilé de "la clarté diffuse de la lagune", ici aussi rêve et réalité s'interpénètrent:

"Un crissement solennel montait des sables, et, comme la frange du tapis qui déborde d'un escalier de rêve, une nappe aveuglante venait se défroisser à mes pieđs même sur les eaux mortes." (p. 105);

la ressemblance des deux chambres va jusqu'à l'emploi des mêmes expressions et images: de même que, dans la chambre des cartes, Aldo se laisse glisser dans "un sommeil peuplé de mauvais songes" (p. 77), la chambre de Vanessa le plonge dans "un sommeil hanté de mauvais songes" (p. 163); à propos de la salle des cartes, Aldo conclut:

"Je sombrais avec elle, debout, comme un épave gorgée du silence des eaux profondes" (p. 33).

— image qui sera appliquée à Vanessa dans sa chambre:

"je sombrais avec elle dans l'eau plombée d'un étang triste, une pierre au cou." (p. 164);

on a l'impression que Vanessa matérialise l'une des présences de l'Ailleurs qui aimantaient Aldo dans la chambre des cartes. D'ailleurs, Vanessa apparaît parfois comme un personnage-fantôme: tout d'abord, elle procure à Aldo une "délectation lugubre" (p. 164); quand elle se réveille au milieu de la nuit, une sorte de mystère insolite l'enveloppe:

“... les traits très légèrement exténués au lumières, elle paraissait lasse et pâle, sérieuse, toute recouverte d'un songe qui 
donnait trop à penser, et la lumière immobile de la lampe ne me rassurait pas. Une fois sa voix s'éleva, bizarrement impersonnelle, une voix de médium ou de somnambule, qui semblait en proie à l'évidence d'un délire calme." (p. 165)

Les passages qui la concernent abondent en expressions ambiguës et imprécises: tantôt elle est comparée à un de "ces anges cruels et funèbres qui secouent leur épée de feu sur une ville foudroyée" ( $p$. 167); tantôt elle se repose "comme vidée de son sang, la tête fauchée par un sommeil sans rêves" (p. 168); tantôt elle parle "comme si quelqu'un d'autre soudain - un esprit d'évidence et de ténèbres - eût parlé par sa bouche"' (p. 254).

Ceependant, beaucoup plus que Vanessa, une autre présence intrigue particulièrement dans cette chambre: celle d'un mort. Le tableau accroché au mur, portrait de Piero Aldobrandi, évoquant l'assaut des Farghiens contre Orsenna, ce tableau bizarrement troublant, qui, pour devenir un élément complètement fantastique, ne demande qu'à s'animer, est une présence presque vivante, aux limites du surnaturel, dans cette chambre vide; avant même d'avoir jeté un regard sur la toile, Aldo avait déjà senti une gêne inexplicable; en la découvrant, il la décrit comme s'il voyait non la peinture, mais Piero Aldobrandi lui-même:

"Et je compris soudain quelle gêne j'avais senti peser sur moi dès mon entrée dans la chambre et tout au long de mon entretien avec Vanessa. Il y avait eu un tiers entre nous. Comme le regard qu'aimante malgré lui par l'échappée d'une fenêtre un lointain de mer ou de pics neigeux, deux yeux grands ouverts apparus sur le mur nu désancraient la pièce, renversaient sa perspective, en prenaient charge comme un capitaine à son bord." (p. 106);

surgie d'une lumière incandescent - "l'ensoleillement d'une aprèsmidi étincelante" (p. 106), "une riche fourrure de flammes", "un buisson ardent" ( $p$. 107) -, la figure peinte de Piero Aldobrandi est à la limite même du réel et du surnaturel; elle ne sort pas de sa toile, mais arrive presque à s'animer là-dedans — et le mot de "surnatu. rel" intervient dans la description:

"le sourire inoubliable du visage qui jaillissait comme un poing pendu de la toile.. semblait venir crever le premier plan du tableau. (...) le visage tendu par une vision secrète était l'emblème d'un surnaturel détachement." (p. 107). 
Ce tableau, que transcende le passé en rendant réel et présent un élément qui appartient au passé, transgresse aussi le présent en offrant une vision prophétique de l'avenir: la bataille entre le Farghestan et Orsenna aura lieu de mouveau, et le cataclysme du Tängri que le tableau annonce viendra encore une fois "faire grésiller ses laves dans la mer" (p. 107): quand le "Redoutable" arrive près du volcan, celui-ci est "mystérieusement ranimé" (p. 213).

Une troisième chambre close, avec à peu près les mêmes caractéristiques des deux autres, est la cabine de Marino sur le Redoutable. Toutefois, ici on n'a pas affaire à des fantômes ressuscités, mais à une présence invisible, "plus lourde que nature" d'un personnage vivant: celle de Marino:.

“ la présence de Marino reflua jusqu'à me fermer les yeux (.. ) aussi extraordinairement intime que celle de la momie dont on détache les bandelettes." (p. 183);

c'est surtout l'image de la momie qui crée l'abiance fantomatique; Al. do se sent "mal à l'aise dans cette pièce songeuse" ( $\mathrm{p}$. 184), où les objets prennent peu à peu possession de lui, jusqu'à ce qu'une image les ranime - exactement comme dans les autres chambres vides; le pouvoir de la pièce sur Aldo est qu'il ne peut s'empêcher d'avoir des visions fantomatiques qui se confondent avec les objets réels:

“. . un moment, le regard englué, je plongeai mes yeux dans son eau grise, et il me sembla que des images toutes pareilles, une infinité d'images à la superposition exacte s'effeuillaient, glissaient indéfiniment l'une sur l'autre à toute vitesse sous mes yeux comme les pages d'un livre, comme la tranche des Instructions nautiques sous mon doigt." (p. 185)

Et ici aussi une prophétie s'élève: quand Aldo part, il referme la porte de la cabine de Marino "du geste précautionneux dont on referme la chambre d'un mort" (p. 185); deux chapitres plus loin, Marino sera un mort.

À côté de ces figures de "fantômes", de "génies", d'anges funèbres" et de "momies", il y a une autre sorte d'images surnaturelles, appartenant au domaine du fantastique enfantin, qui interviennent parfois: Fabrizio compare le Farghestan à Croquemitaine (p. 63); Vanessa, dans une métaphore, traite Aldo de Cendrillon (p. 80); l'envoyé du Farghestan apparaît comme une figure de conte de fées qui fait penser au génie de la lampe - "une apparition silencieuse ensorcelée" ( $p$. 230); le récit de la Croisière progresse comme "un conte de fées" (p. 220). 
Ces figures fantastiques, de par leur seule allusion - elles ne sont pas très nombreuses - rendent plus vastes les dimensions du thème de l'insolite dans le récit gracquien: nous sortons du fantomatique produit par une imagination individuelle - celle d'Aldo pour pénétrer dans le domaine de l'imaginaire collectif de l'univers fantastique. Il ne faut pourtant pas oublier que ces figures n'apparaissent qu'au niveau de la métaphore et de la comparaison, elles aussi: l'aspect d'irréalité du roman se trouve plutôt dans la création d'une atmosphère particulière que dans l'intrigue proprement dite. La facilité avec laquelle réel et fantastique sont mis en relation dans ces images révèle aussi, tout en accentuant l'irréalité du vécu, l'irruption de l'Ailleurs dans la vie quotidienne; le passage du fantomatique au fantastique jette un pont entre ces deux thèmes dans le récit (12).

\section{b) Le mystérieux}

On pourrait dire que l'univers du Rivage des Syrtes est un univers mystérieux par excellence: à chaque instant une énigme est posée - et l'auteur ne nous en donne pas toujours la réponse; le climat de mystère est établi progressivement, et le résultat est l'impression de "suspens" que l'on sent. Il ne s'agit pas d'événements mystérieux qui arrivent - d'ailleurs, il n'arrive presque rien! - mais d'un mystère qui plane et qui s'élève des choses, des sensations, du paysage, de la nature, des personnages, du vide même, du rien même, sans qu'il y ait une cause ou une explication logique; sur ce monde endormi, sur 1"irrémédiable décadence" de ces terres pourries, sur ce chemin vers la mort, planent mystérieusement des signes de vie.

Il s'agit, au début, d'un mystère imprécis; déjà dans la deuxième page du livre, le mot "secret" apparaît; il sert à établir un curieux paradoxe avec la description de la vie sans attraits, aux journées égales, qu'offre Orsenna. Il surgit quelques lignes après cette description et semble sortir du néant: il n'a pas de liaison avec ce qui le précède, ni avec ce qui s'ensuit; mais il annonce une première rupture: bientôt on apprendra qu'Aldo a sollicité un poste dans une province lointaine (cf. p. 9). Si dérisoire que ce départ puisse parâ̂tre au début, il prend peu à peu les couleurs du mystère:

"mon exil était plus sérieux et plus lointain qu'il n'avait d'abord paru; chacun sentait que la vie pour moi s'apprêtait à vraiment changer" (p. 11).

(12) - Pour une étude plus approfondie du fantastique dans ce roman, voir notre dissertation de Maîtrise déjà citée, pp. 174 à 186 . 
Le voyage d'Aldo débute par l'opposition entre "ville familière" et "destination ignorée" (ibid.): une rupture a eu lieu; seulement, le mot "ignorée" indique qu'il ne sait pas encore en quoi elle consiste; c'est l'inconnu qui fait irruption dans la vie quotidienne. Cet inconnu se revêt bientôt d'un vague mystère: parmi ses affaires, Aldo trouve une enveloppe scellée contenant les "ordres secrets" de la Seigneurie - ce qui éveille la curiosité du lecteur. De plus, c'est le mot de "mystère" qui ouvre le récit de l'état de guerre entre Orsenna et le Farghestan (cf. p. 12); tout contribue à entourer l'aventure future d'Aldo d'un climat vaguement mystérieux. Les éléments du paysage décrit tout au long du voyage vers les Syrtes prennent, à mesure que l'on s'approche de l'Amirauté, un caractère de plus en plus suspect; de vagues pressentiments remplissent l'esprit d'Aldo; il sent que quelque chose va arriver, sans savoir quoi, ni pourquoi:

je me baignais pour la première fois dans ces nuits inconnues d'Orsenna, comme dans une eau initiatique. Quelque chose m'était promis, quelque chose m'était dévoilé; j'entrais sans éclaircissement aucun dans une intimité presque angoissante, j'attendais le matin, offert déjà de tous mes yeux aveugles, comme on s'avance les yeux bandés vers le lieu de la révélation" (p. 18).

Dans ce passage, éléments inquiétants et éléments prophétiques alternent: d'un côté, "nuits inconnues" "sans éclaircissement aucun" "intimité angoissante" et "mes yeux aveugles" révèlent un mystère caché, une peur inexplicable; d'un autre côté, "quelque chose m'était dévoilé" "j’attendais le matin" et "le lieu de la révélation" évoquent la nuit de la Croisière et l'approche du Tängri. L'inquiétude que le paysage suscite est renforcée par "l'intimité suspecte et pénétrante de la pluie" (p. 18), par "le silence suspect du paysage" - signalons, en passant, la répétition du mot "suspect" - et par "l'impression de suspens insolite que communiquaient ces intervalles inégaux” (p. 19), jusqu'à devenir une sensation de présence cachée:

\footnotetext{
"Quelque chose s'étouffait derrière ce brouillard de terrain vague, comme une bouche sous un oreiller." (p. 19)
}

Une fois dans les Syrtes, cet élément mystérieux prend forme peu à peu: il s'agit de signes de vie qui émanent inexplicablement de la mort même; dans ces parages qui attendent la mort, où rien ne se passe, quelque chose s'ébauche et prend corps. L'Amirauté est plongée dans un sommeil inquiet:

“ il montait de cette naive activité villageoise une inquiétude et un appel. Un rêve semblait peser de toute sa 
masse sur la somnolence de ces allées et venues $($.$) ; . . je$ sentais monter en moi cette fascination d'étrangeté.." (p. 28) (13).

Une manace semble peser sur cette sécurité; le mot revient deux fois: dans "étendue menacée" (p. 28) et dans "approche menaçante" (p. 76). La Forteresse, avec ses "voûtes aux échos inquiétants" ( $p$. 22), devient bientôt pour Aldo une "terre secrète" où il découvre "un trésor enseveli" (p. 35): la chambre des cartes, ce "mystérieux centre de gravité"; la fascination que le mystère enfermé dans cette chambre exerce sur Aldo est telle qu'elle finit par remplacer chez lui jusqu'au plaisir physique:

"Jétonnais Fabrizio en refusante jusqu'aux plaisirs faciles et aux amours d'une heure qu'il allait chercher presque chaque semaine à Maremma. Je n'en avais plus besoin." (p. 134) .

Il y a là un secret - cela ne nous est pas caché: on retrouve ce mot plusieurs fois dans la description - et Aldo le cherche; c'est peut-être dans ce secret que se trouve la source de l'inquiétude qui menace ce sommeil engourdi.

A Sagra, au milieu des "débris médiocres" (p. 71), paradoxalement mort et vie se confrontent; l'agonie de la nature suggère un obscur désastre évoqué par la lutte insolite de ses éléments, qui semblent se ranimer au contact même de la mort, et "font bondir au coeur comme un sentiment panique de la permanence de la vie" (p. 71) Au milieu de cette mystérieuse confrontation, le secret crerché par Aldo se précise, se matérialisant dans la "silhouette insolite" d'un bateau, "cerné de mystère" Aldo a raison: quelque chose n'est plus dans l'ordre (cf. p. 72). Et tout à coup, ce mystère insolite qui jette des pierres dans les eaux tranquilles d'un engourdissement agonisant, prend finalement un nom:

"Je ne pouvais plus me dissimuler l'importance excessive que commençait à prendre tout ce qui, de près ou de loin, se rapportait au Fargestan ( ). Des brumes trop rassurantes s'étaient dissipées. Il y avait une côte devant moi où pouvaien1 aborder les navires, une terre où d'autres hommes pouvaient imaginer et se souvenir." (pp. 73-74).

Mais le fait d'avoir pris un nom ne veut pas dire pour autant que l'énigme est résolue, que le mystère est levé; bien au contraire,

(13) - C'est nous qui soulignons. 
ce nom est en lui-même une énigme. L'atmosphère qui l'entoure, chaque fois qu'il intervient dans la narration, est de plus en plus imprécise et troublante, comme s'il s'agissait d'un pays de songe ou de danger. Son introduction s'accompagne déjà d'une curieuse suspension:

"On sait peu de chose e dans la Seigneurie sur le Farghes$\tan (\ldots)$. - on ne souhaite guère en savoir davantage." (pp. 12-13);

la deuxième allusion a lieu dans la chambre des cartes: des mots tels que "magique", "inconnus", "terre sainte", "obsédantes" font de ce pays un vrai foyer mystique (cf. p. 32); l'insistance sur la distance qui sépare le Farghestan des limites d'Orsenna - "très au-delà, prodigieux d'éloignement" - intensifie le mystère et la curiosité que provoque ce pays, car elle met l'accent sur la difficulté d'y accéder et sur le caractère d'inconnu dont il se revêt. La troisième fois, c'est Aldo qui prononce ce nom devant Marino: le mot tombe dans un "silence de catastrophe" comme s'il venait troubler ce silence; pour Marino, il s'agit d'un mot-tabou: il en a peur; l'interdit de la ligne de frontière semble s'étendre jusqu'au langage:

$$
\text { "C'ext un nom qui n'a guère cours (ici)" (p. 45). }
$$

Dans cette progression, le mot lui-même finit par se magnétiser, comme chargé d'une tension électrique; lorsque Belsenza l'articule, Aldo se sent "parcourir d'une onde légère" (p. 91); l'impression produite par le seul nom est tellement forte qu'on évite même de le prononcer: à Maremma, il est remplacé par "là-bas"; on en parle comme d'un lieu où il ne se passe que des choses bizarres (cf. pp. 93 et 94)

Entretemps, ce mystère, doté maintenant d'un nom, fait son entrée dans la vie quotidienne à Maremma, en y apportant tout ce qu'il comporte d'insolite, de nouveauté, et remet en question tout un système: la "fièvre qui minait la ville" augmente de plus en plus (cf. p. 143); les prophètes sinistres - "des oiseaux de mauvais augure" se multiplient (cf. pp. 154 et 155); durant la scène de la jeune fille fouettée "quelque chose d'insolite, dans ce silence de tombe, arrêtait les plaisanteries habituelles" (pp. 155-156). L'église de Saint-Damase, "la rebelle incorrigible" cette étrange église hérétique "célèbre. par la suspicion opiniâtro attachće à la liturgie et aux rites qu'elle abritait" (p. 172), est l'un des centres de divulgation des "vapeurs suspects" qui envahissent les rues; des tapisseries qui ornent les murs, représentant "une nativité plus menacée" (p. 174), aux mystérieuses paroles du prêtre (pp. 176 à 179), tout y annonce le danger imminent et la destruction du sommeil et de la sécurité. Le sermon 
s'inscrit dans le cadre du symbolisme chrétien (c'est un sermon de Noël), mais les symboles prennent une signification nouvelle: ce n'est pas la lumière en soi, ni la naissance elle-même, ni le Christ venant de naître qui en font l'objet, mais l" "étoile bougeante et muette", témoin de la lumière future, "l'heure de l'angoissant passage"; ce sermon insolite explique moins l'advenu qu'il ne renvoie à l'avenir dont il célèbre l'acceptation. Il s'agit à la fois d'une oraison funèbre exaltant "la destruction et la mort" - et d'un chant de joie - apportant la nouvelle d'une naissance, quoique "ténébreuse"

Le mystérieux se manifeste aussi à travers les personnages du roman. Vanessa, dès son introduction dans l'histoire, est présentée comme un personnage énigmatique: surgie du silence d'un "lieu magique" (14), elle n'est définissable que par des expressions imprécises ou antithétiques tels que: "une jeune fille ou une très jeune femme" (p. 51); "sa voix. trahissait une calme exaltation" (p. 53); "dans ses yeux passait le reflet trouble des mers lointaines" (ibid).

L'envoyé du Farghestan est lui aussi un personnage mystérieux; son apparition au milieu des ruines de Sagra frappe par son caractère vaguement fantomatique; dans la chambre d'Aldo cet inconnu surgit comme la personnification même du mystère qui émane du Farghestan: il apparaît dans la "nuit silencieuse", surgi on ne sait bien d'où; son portrait révèle des caractéristiques mystérieuses, et, à mesure qu'il parle, l'énigme qu'il instaure augmente et se complique, au lieu d'être résolue (cf. pp. 224 à 230). Quand il s'en va, il laisse derrière lui une impression d'hallucination et de présage sinistre:

"La lente, la silencieuse ondulation de reptile qu'il avait eue pour sortir de l'ombre et pour s'y évanouir, la fascination qu'avaient exercé ( $\mathrm{sic}$ ) sur moi ses yeux et sa voix, et l'heure très tardive, m'auraient donné à croire à une hallucination ( .). Une note sinistre résonnait avec les derniers mots de l'étranger dans mon esprit vide" (pp. 237 et 238)

C'est cependant un troisième personnage qui mérite une mention particulière ici: sans aucune autre fonction que d'être un noyau d'insolite, une jeune femme exerce, dans la fête de Vanessa au palais Aldobrandi, un étrange pouvoir de fascination sur Aldo; il ne la voit pas, il ne l'entend pas, il la sent:

(14) - Il s'agit des Jardins Selvaggi (cf. p. 50) 


\begin{abstract}
“ j'éprouvai soudain distinctement, comme un souffle sur la nuque, le sentiment d'une présence plus alertée et plus proche." (p. 88);
\end{abstract}

ses yeux cachent le mystère des profondeurs obscures (cf. p. 89); son portrait frappe doublement: d'une part, par le recours exagéré aux comparaisons et aux métaphores: dans l'espace de vingt lignes, les huit propositions qui la peignent contiennent quinze images de ce genre, le mot "comme" apparaissant sept fois (ibid); ce procédé nous semble relever de la difficulté de définir l'objet. D'autre part, ces images ont un caractère tout à fait étrange, à la limite même de la cohérence: si elles ne sont pas complètement surréalistes parce qu'il n'y a pas d'interférence sémantique du plan métaphorique sur le plan littéral (15), elles rapprochent tout au moins deux réalités assez eloignées pour ne pas être considérées comme des anomalies sémantiques (16). En voici des exemples:

"La bouche aussi vivait comme sous les doigts, d'un tremblement rétractile, nue comme un petit cratère de gelée marire ( ) Comme on raccorde dans la stupeur les anneaux d'un serpent emmêlé, s'organisait par saccades autour de cette tête de méduse une conformation bizarre." (p. 89).

Ce personnage semble représenter la matérialisation même du mystère indéfinissable qui plane sur le monde du roman depuis le début, et qui finalement ne se rapporte ni au Farghestan, ni à la guerre imminente, mais à la seule constatation de l'existence de l'irrationnel dans le réel.

Le mystérieux s'introduit aussi dans certaines sensations qui se manifestent chez les personnages d'une façon inexplicable ou, tout au moins, inattendue: tantôt des bruits isolés, qui prennent un caractère insolite du fait qu'ils se détachent sur un fond de silence absolu, résonnant comme des pressentiments; tantôt des frémissements provoqués par une bizarre sensation de froid, qui accompagne certains événements ou certaines apparitions plus ou moins étranges. Cependant, c'est surtout des sensations magnétiques qu'on voudrait parler ici: on a déjà voulu considérer Le Rivage des Syrtes comme un système de polarité, avec des pôles négatifs et des pôles positifs (17) Ef-

(15) - Une des caractéristiques du récit surréaliste, établies par L. Jenny à partir de l'analyse du Poisson Soluble d'A. Breton; dans "La surréalité et ses signes narratifs" in Poétique 16, Paris, Seuil, 1973, p. 502.

(16) - A ce sujet, voir l'article de $\mathrm{T}$ Todorov, "Les anomalies sé. mantiques" in Langages $\mathrm{n}^{0} 1$. Paris, Larousse, 1966.

(17) - Cf. Jean Baudry, "Julien Gracq, poète-romancier", in Revue des Sciences Humaines, fasc. 88, oct.-déc. 1957, p. 471. 
fectivement, on a souvent l'impression qu'une sorte de magnétisme s'exerce sur Aldo, que certains lieux ou personnages s'avèrent condensateurs d'énergie, et établisent autour de lui un courant électrique de repulsion ou d'attraction. Par exemple, à Sagra, en marchant vers le sud, le personnage observe:

“... un magnétisme secret m'orientait par rapport à la bonne direction." (p. 69).

En roulant vers Maremma, il aperçoit derrière lui la forteresse de l'Almirauté et devant, à l'horizon, le Farghestan: un courant électrique s'établit entre l'une et l'autre:

".. il me semblait sentir ces deux pôles, autour desquels maintenant oscillait ma vie, se charger d'une subtile élictricité." (p. 139)

Le même phénomène se produit dans l'église Saint-Damase:

"... le reste de la nef était très sombre, mais il en venait cette communication magnétique et presque tactile, pareille à l'air aspiré tremblant au-dessus d'une route chaude, qui monte d'une foule communiant dans l'extrême ferveur." (p. 174)

Le Tängri, ce volcan du Farghestan auquel nous avons déjà fait plus d'une allusion, est le lieu condensateur et redistributeur de forces par excellence, autour duquel tout se magnétise; à sa vue, tout le navire se charge d'électricité (cf. p. 206) à tel point qu'Aldo observe:

"Un charme nous plaquait déjà à cette montagne aimantée." (p. 216)

Ce courant électrique va jusqu'à atteindre Orsenna, où "de grandes lignes de force inscrites dans le sol. par son histoire se rechargeaient d'une électricité active" (p. 292).

Parmi les personnages qui diffusent cette mystérieuse force d'aimantation, Vanessa est en premier chef; dès son entrée dans le récit elle se révèle un personnage catalyseur d'énergie qui vient "charger d'une brusque tension" la vie d'Aldo (cf. p. 75); une sorte de courant éléctrique semble lier ces deux personnages:

couché contre elle, il me semblait. sentir glisser avec nous l'épanchement ininterrompu d'un courant rapide" (p. 162) 
D’autre part, Aldo se sent également, parfois, "branché” à Marino:

“... par ce bateau lancé qui vibrait sous nos pieds, nous communiquions dans les profondeurs." (p. 58)

On dirait qu'il s'agit des deux pôles, l'un positif (Vanessa), et l'autre négatif (Marino), entre lesquels Aldo, cette "machine électrique à haute tension" (18), reçoit et renvoie des influx magnétiques.

Fantomatiques ou mystérieuses, ces manifestations de l'insolite viennent déranger le réel en faisant intervenir l'irrationnel dans un un monde logique; elles provoquent l'imagination et la prédispose à la rupture des lois naturelles; elles en avertissent l'individu et suscitent son désir: quelque chose doit se passer

Et c'est justement ce désir qui, dans l'univers du roman, va provoquer les événements. Avant que ceux-ci ne se réalisent, ils ont déjà été vécus en rêve et dans l'attente de la réalisation de ce rêve, et peu à peu ils deviennent: l'imaginaire devient réel; ce qui était craint, ce qui était désiré, ce qui était attendu, ce qui était vécu de forme latente dans l'imaginaire, devient vrai et atteint finalement la même force de présence que le réel; ce qui était rêverie statique devient imagination dynamique

C'est par ce jeu de l'imagination que l'événement central du livre - la provocation au Farghestan qui déclenchera la guerre se réalisera: la transgression de la frontière d'alarme, suscitée chez Aldo par les fantômes qui hantaient la chambre des cartes et qui exerçaient sur lui une attraction irrésistible, sera finalement accomplie:

"Le Farghestan avait dressé devant moi des brisants de rêve, l'au-delà fabuleux d'une mer interdite; il était maintenant une frange accore de côte rocheuse, à deux journées de mer d'Orsenna." (p. 199):

On dirait qu'Aldo a toujours l'intuition de ce qui va arriver, à partir de deux forces qui se recoupent: celle du monde extérieur et celle du monde intérieur: les événements sont suscités d'une part par le désir intense du personnage et d'autre part par l'appel qui vient du monde extérieur: le magnétisme des Syrtes, le pouvoir de fascination des lieux, la force d'aimantation du Tängri. Ce double mouvement provoque l'intrigue, que se situe au point de rencontre du désir subjectif avec le réel objectif. L'imaginaire n'est donc pas purement

(18) - J. Gracq, André Breton. Paris, Corti, 1948. 
phantasmé, il est l'embryon du réel: dans la dynamique de l'imagination gracquienne, "l'imaginaire est ce qui tend à devenir réel" (19)

C'est la notion de hasard objectif, telle qu'elle a été conçue par les surréalistes, qui rend compte de cette dynamique: pour chaque rencontre, il y a toujours deux séries causales que se croisent et se rejoignent: celle du désir subjectif du personnage et celle du déterminisme du réel objectif. Pendant la croisière, Aldo d'interroge sur lees "indéchiffrables coïncidences" ( $p$. 213) qui ont mené sa vie - ce qui nous fait revenir en arrière pour examiner de plus près les diverses "apparitions" dans le récit, chacune correspondant à un degré dans l'échelle d'événements qui aboutit à la croisière.

L'apparition du "bateau-fantôme" (p. 41) est une première rencontre coïncidente: jusqu'alors, les signes d'une possible rupture de l'ordre n'existaient que dans le désir subjectif d'Aldo: en regardant la mer, il attendait un signal, il rêvait d'une "voile désirée" (p. 36); le déterminisme causal du réel objectif vient rejoindre ses désirs: on saura plus tard que le Farghestan avait déjà envoyé un espion à la mer des Syrtes; ainsi, l'objet désiré, cette "voile naissant du vide de la mer" (ibid.), se matérialise tout à coup devant lui. Subjectivité et objectivité se croisent, les lois déterministes du réel objectif répondent aux appels du désir subjectif; celui-ci devient réel.

Ce même mouvement peut être observé dans la rencontre du bateau et de l'espion à Sagra: devant cette "apparition fiévreusement espérée" ( $p$. 72), rendue réelle par le causalité déterministe (c'était le bateau que Vanessa cachait, parce qu'il n'était pas régulièrement immatriculé), Aldo se réjouit: il sent "monter au coeur. une espèce d'épanouissement intime" ( $\mathrm{p}$ 22)

Vanessa surgit devant Aldo au moment où il songeait au signe d'appel qu'elle lui avait adressé (cf. pp. 74, 75 et 77). L'épisode de l'arrivée des instructions de la Seigneurie obéit à la même dynamique: étrange coïncidence, qui rejoint les désirs d'Aldo en donnant "une consistance et une forme au fantôme qui (le) fascinait déjà" (p. 138); on apprend plus tard qu'il ne s'agissait que d'une pièce du système causal logique qui régit les événements du récit: la volonté de Danielo. L'absence "accidentelle" de Marino à l'Amirauté, qui a permis à Aldo de commander la patrouille de nuit, et de concretiser alors tous ses désirs, a été provoquée par Danielo. Et finalement, la guerre, ce mot tellement craint et tellement désiré dans l'inconscient des personnages, trouve une réponse dans les trois coups de canon: le monde "réel" du Farghestan attendait déjà la provocation; comme disait Bre-

(19) - A. Breton, La Clé des Champs. Paris, Pauvert, 1967, p. 108. 
ton pour définir le hasard, c'est la "nécessité extérieure" qui s'est frayé "un chemin dans l'inconscient humain" (20).

Le Rivage des Syrtes apparaît maintenant comme une succession de hasards inconsciemment souhaités qui s'enchaînent en déréglant progressivement le réel et qui finissent par croiser la nécessité extérieure: le cercle se ferme peu à peu, et finalement il n'existera qu'une seule possibilité pour Orsenna: l'invasion du Farghestan, qui entraînera la destruction fatale de l'ancien système. Le réel objectif du Rivage des Syrtes n'est donc que la projection d'un imaginaire dans le monde concret; dans la rencontre des deux se trouve la vraie réalisation du roman.

(20) - L'amour Fou. Paris, Gallimard, 1937, p. 32. 